Between 2000-2015 Venezuela witnessed a 365\% increase in malaria cases followed by a $68 \%$ increase $(319,765$ cases) in late 2017. The latest figures have surpassed 600,000 malaria cases with a prediction to reach 1 million by the end of 2018 . Measles and diphtheria have recently re-emerged after a progressive interruption of the national immunization program, with vulnerable indigenous population being particularly affected. In response to Venezuela's rapidly decaying situation, a massive population exodus is ongoing towards neighboring countries causing a spillover of diseases.

Discussion: Action to halt the spread of vaccine-preventable diseases within Venezuela is a matter of urgency for the country and the region. Global and hemispheric health authorities should urge the Venezuelan government to allow establishing a humanitarian channel to bring relief.

Prehosp. Disaster Med. 2019;34(Suppl. 1):s5-s6

doi:10.1017/S1049023X1900030X

\section{Surge Capacity Planning to Inform the Need for International and Domestic Emergency Medical Team Deployments Following a Severe Wellington, New Zealand Earthquake \\ Mr. Charles Blanch ${ }^{1,2}$, Dr. Emma Lawrey ${ }^{1,3}$ \\ 1. New Zealand Medical Assistance Team, Auckland, New Zealand \\ 2. Ministry of Health, Wellington, New Zealand \\ 3. Auckland City Hospital, Auckland, New Zealand}

Introduction: Wellington, New Zealand has a significant earthquake risk with unique response challenges posed by its geography and limited road, rail, and sea access. In 2014, the World Health Organization (WHO) Emergency Medical Team (EMT) initiative published minimum and technical standards for EMTs in response to failures by responding teams to deliver appropriate and ethical clinical care during a number of disasters (Norton, 2014). The initiative has evolved to develop national and International EMTs in addition to a support capacity building within Ministries of Health to better coordinate clinical capacity during an emergency.

Aim: Over the last two years, the WHO EMT Coordination Cell (EMTCC) course has trained over 300 health personnel globally to coordinate clinical surge capacity using a three-step Impact Assessment, Needs Assessment, and Tasking process informed by disaster epidemiology and mass casualty ratios.

Methods: EMTCC planning methodology was applied to the "Wellington Earthquake National Initial Response Plan" (MCDEM, 2017) to develop a Health Action Plan for a significant Wellington earthquake. Known earthquake impact modeling for injuries was applied against predicted capacity in receiving hospitals in the affected region, and the ability to transfer patients nationally to determine unmet response needs. EMT minimum standards and operational insights from recent disasters were then used to determine the number of EMTs required for optimal tasking.

Discussion: The surge planning methodology provided a theoretical framework for national and local health emergency management staff to engage with clinical colleagues. This allowed likely EMT assistance to be pre-planned, which facilitates further planning with national and local emergency management, border, and registration agencies for rapid entry into NZ, including onward transport and logistical support. While injury treatment ratios had to be refined to reflect NZ context, the methodology proved useful for Ministries of Health to pre-identify the need for international assistance in national emergencies.

Prehosp. Disaster Med. 2019;34(Suppl. 1):s6

doi:10.1017/S1049023X19000311

The Importance of Enforcing Road Safety Laws to Reduce Road Traffic Collision (RTC) Occurrence and Fatalities in Nigeria

Dr. Oluwafunbi Awoniyi ${ }^{1,2}$, Dr. Michael Molloy ${ }^{1,3}$, Dr. Alexander Hart ${ }^{1,2}$, Amalia Voskanyan ${ }^{1,2}$, Dr. Ritu Sarin ${ }^{1,2}$, A Prof. Gregory Ciottone ${ }^{1,2}$

1. BIDMC Fellowship in Disaster Medicine, Boston, United States

2. Department of Emergency Medicine, Beth Israel Deaconess Medical Center, Boston, United States

3. University College Dublin, Belfield, Dublin, Ireland

Introduction: Road Traffic Crashes (RTC) are one of the most preventable causes of death worldwide, yet are the number one cause of death in Nigeria. In March 2010, the United Nations General Assembly launched "The Decade of Action for Road Safety (2011-2020)" to "reduce road traffic deaths and injuries by $50 \%$ by $2020 . "$

Aim: To analyze trends in RTC and deaths in relation to current road safety laws in Nigeria, and possible future interventions.

Methods: Annual reports from 2013-2017 were obtained from the Federal Road Safety Corps (FRSC) of Nigeria. These reports were analyzed for trends in RTC, deaths, and reported causes to find areas of possible improvement.

Results: The number of RTC and deaths declined yearly from 2013-2017. Crashes decreased from $23.4 \%$ in 2013-2014 to $6.2 \%$ in 2015 , to $0.4 \%$ in 2016 , and then increased to $3.2 \%$ in 2017. Results showed that fatalities from RTC in 2013-2014 decreased by $8.4 \%$, then by $9.3 \%$ in 2015 , and by $7.1 \%$ in 2016 , but had a 1.3\% increase in fatalities from 2016-2017. Analysis showed that speed violations (SPV) were the top cause of RTC. These had a decrease in the number of crashes from 5,495 (32\% of RTC) in 2013, to 3,496 (29\%) in 2014, to 3,195 (26.5\%) in 2015. They then increased to 3,848 (33.9\%) in 2016 and to 4,840 (44.1\%) in 2017. There was a decline in reports of RTC caused by driving under the influence (DAD) from $1 \%$ in 2013, to $0.8 \%$ in 2014 , and $0.5 \%$ in 2015 and 2016 .

Discussion: Current road safety laws have been effective in decreasing the total number of RTC and deaths. While certain laws such as those regarding DAD have been effective, other laws such as speed limits have been less successful and may require further changes in legal codes and/or enforcement.

Prehosp. Disaster Med. 2019;34(Suppl. 1):s6

doi:10.1017/S1049023X19000323 